\title{
Visual Data Analysis Methods Using OpenCV Programs to Evaluate Walking and Falling with a Japanese Walking Support System
}

\author{
Shinji Kawakura* \\ National Institute of Advanced Industrial Science and Technology (AIST), Department of Information \\ Technology and Human Factors, Robot Innovation Research Center 1-1-1 Umezono, Tsukuba, Ibaraki \\ 305-8560 Japan. \\ * Corresponding author. Tel.: +81-80-9781-1535; email: s.kawakura@aist.go.jp \\ Manuscript submitted June 26, 2017; accepted August 20, 2017. \\ doi: 10.17706/ijbbb.2018.8.1.20-31
}

\begin{abstract}
In recent years, researchers and engineers have come together to develop diverse, applied, and practical sensing systems to solve the difficulties faced in the development of advanced support systems, technical teaching, and safety issues for physically challenged and elderly people. Following a sequence of studies developing promising systems that address a number of nursing challenges, the purpose of this prospective research was to develop effective systems and demonstrate their accuracy and utility for the aforementioned people. In this kinematic investigation, we develop a physical analysis system, which uses two video cameras to obtain visual data of physically challenged and elderly people from two directions (the subject's front and left). These systems use the OpenCV 2.4.9 package, including the library and header files, and programs originally written in Visual $\mathrm{C}++$. This study examines the qualitative and quantitative characteristics and the unique parameters of (1) the main shaft (the principal axis of inertia) of the subject and the walking support system to highlight the differences between two frames using binary video data, and (2) coordinate values of characteristic points that are set automatically. Finally, we present the output values for the physical measurements obtained from various viewpoints. In future, these methods could be of practical use in providing alternative directions for developers and care managers to assess and treat users' conditions in both outdoor (e.g., playgrounds for the elderly) and indoor settings (e.g., hospitals).
\end{abstract}

Key words: Falling with patients and the elderly, human engineering methodology, OpenCV, safe technology measurement, walking support system.

\section{Introduction}

In recent years, a variety of system development techniques have been created and instituted across diverse fields including disability, rehabilitation, and sports [1]-[5]. Previous research in several scientific fields has executed electric walking supporting systems (Rollator) and simple, non-electric systems, the tracking of characteristic points, and the drawing of stick pictures [6]-[13]. However, limited research has examined the gravity points and the main shaft (the principal axis of inertia) of the actor. In addition, targeted achievements concerning living human bodies and non-living humanoid devices have been somewhat limited because of social and ethical issues [5], [13]-[15]. The aim of this study was to analyze visual data using OpenCV 2.4.9-based programs to calculate the parameters of walking support system users [16]]-[18]. Specifically, we calculated the timeline of the inclination of the main (principal) axis, its 
moment, the coordinates of the center of gravity, and the coordinates of characteristic points when the subject walked on a flat surface using the walking support system. Although neither human bodies nor walking support systems are simple structures, we propose using index values as references for measurements, and we evaluated the users' (medical) condition and habituation to the equipment. We also accumulated timeline data from 18 points on the subject's body including 3D-coordinates (the amount of transfer in space), speed, posture (using quaternion values), acceleration, and angular velocity using Perception Neuron (PN); one motion capture system. We have been developing methods for the remote observation of physically challenged people in parallel with contact-type methods (e.g., wearable electronic systems [19], [20]) for many years. These visual methods of analyzing digital data from systems could be useful and provide promising indicators for not only improving users' movements but also revising nursing techniques. Such systems could even save users from sudden or hidden diseases and injuries in outdoor and indoor settings. In future, the results of these investigations could thus help disabled and elderly people, and their care managers.

\section{Theory}

\subsection{Experimental Settings}

To develop advanced support systems, improve technical teaching, and address safety issues for physically challenged people, we have conducted a sequence of studies to develop systems that address a number of nursing challenges. One purpose of this prospective research study is to develop these systems and demonstrate their accuracy and utility. The experimental settings are given below. The various types of walking support systems, which are available for purchase worldwide, differ according to district, climate, and lifestyle in terms of weight, length, and form, which refers to the leaning style, the wheels, the shaft(s), the arm rest (elbow rest), etc. Following preliminary research, we selected a typical Japanese walking support system (Rollator) (Fig. 1) to use in this study, accounting for shape, weight, and size, because the system is commonly used in Japan, where the study was conducted. The official name is the Rollator KA-391 (Paramount Bed Co., Ltd., Japan). The dimensions are width $=660 \mathrm{~mm}$, length $=732 \mathrm{~mm}$, height $=$ 966-1,218 $\mathrm{mm}$ (adjustable), and weight $=12.5 \mathrm{~kg}$, and the shafts are made from aluminum. The points where subjects put their elbows (on the elbow rest) are made of plastic. Subjects can grasp the handle anywhere with their other (non-dominant) hand.

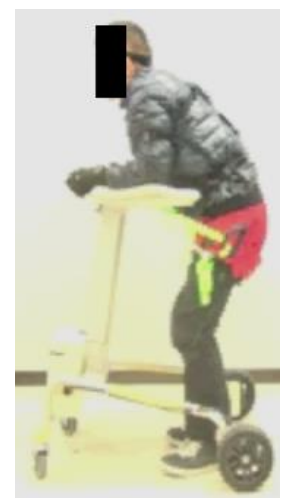

Fig. 1. Walking support system and subject.

First, we categorized various walking support systems used in the fields of nursing welfare, human engineering, car production, and household electrical products based on their form, physical intensities for users, main postures, and operation (manual/electric). Second, we examined the actions of (1) walking straight for $2.0 \mathrm{~m}$ at usual speed and (2) falling in four directions (forward, backward, to the right, and to 
the left), because these are common full-body movements. When simulating a fall, to prevent their body from suddenly falling away from the machine, the user was asked to grasp the handle of the machine and wear a harness, which attached him to the machine at the waist and hips to keep their body from suddenly falling from the machine. The user executed the aforementioned movements five times successively, with 10-second intervals between each trial. However, it was difficult to know whether the characteristics and numbers of tasks or the experiment sets were appropriate or not at that time and in that situation. We selected one Japanese male subject with the appropriate body size and features $(172 \mathrm{~cm}, 63 \mathrm{~kg}$ (these are enough close to the average Japanese male values) and 38 years old) to verify our systems and obtain physical data. According a preliminary interview, the subject fulfilled the criteria of no serious diseases, peculiar habits, or prior relevant careers, such as being an expert in sports or martial arts.

To collect fundamental information about the subject, we used a questionnaire from the Japan Association of Industrial Health and other health organizations, which had previously established reliability. Further, we checked the subject's level of fatigue after each trial, and we evaluated his fitness level, smoking habits, and the occurrence of backache to establish his condition. In line with similar studies in which researchers have measured the motion and position of the subject's bones, but not the muscles, we focused on the right crest of the subject's iliac bone. Previous sensing and tracking of the left and right iliac crests have indicated that the crest (top) of the ilium (the iliac crest) is the superior border of the ilium wing.

For the camera system setup, we selected two common, nonspecific medium format digital video cameras: SONY HDR-PJ790V. We chose these more universal cameras rather than the specialized high-speed cameras used in the fields of human dynamics and sports sciences in consideration of future perspective uses of such measurement systems. The camera sampling rate was $30 \mathrm{fps}$ (frames per second), and the frame size was $1280 \times 720$ pixel (that is, $16: 9$ ), with standard mode settings. The distances from the fixed cameras to the subject, when static (not walking) were 3.0 meters from the side and 1.5 meters from the front (Fig. 2).

After obtaining the visual data, we converted the files into AVI format. The frame size and fps of the visual data were uniformly changed into $320 \times 240$ pixels. This conversion was done to ensure compatibility with a Visual C++ 2010 express-based program and the OpenCV library and header files. Based on findings of past studies, the settings were reasonable for use in the analyses of general nursing workers and other social scientists, directors, and managers to obtain a time series of visual data for diverse analyses of indoor experiments. Although many existing studies use the visual data capturing library, header files, or program codes from OpenCV (ver. 2.4.9) to obtain visual data, we lacked confidence in these methods because of their potential negative impact on recording accuracy. We thus utilized these elements only for the analyses, after the visual data were processed.

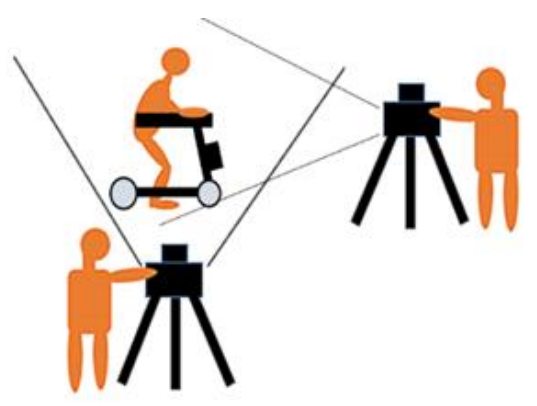

Fig. 2. Camera, walking support system, and subject set-up.

We used a flat surface at the National Institute of Science and Technology (AIST) and consolidated the other experimental field settings as much as possible. We ensured that the subject's clothes were appropriate for the physical demands of the conditions of the visual data analysis. The subject therefore 
wore a black jumper (jacket), casual pants, and shoes, similar to the casual, unspecific fashions in Japan. The clothing was thin and tight to minimize the chances of the sensor nodes sliding. The background walls and floor of the space were a natural white color, and one white door was located on the right side of the wall. However, the white soles of the shoes were did not affect the visual analysis substantially. Using computer program-based processes, we confirmed the adequacy of the color contrast. As the clothing contained no protruding items or accessories, it was appropriate for the experiments. We conducted visual analyses by calculating the differences between two successive frames of the visual data.

\subsection{Settings of Experiment}

We analyzed the visual data (in regulated AVI format) using original programs written in Visual C++ 2010 Express (Microsoft Corporation). The following paragraphs explain the theories underlying our image analyses. We used Shi-Tomasi's method and the function cvGoodFeaturesToTrack in the OpenCV library to execute an automatic search for the characteristic points in directed pictures. Although the Harris method can be used to change parameters, it was not used in this study because of these characteristics. The two methods are mathematically similar. When focusing on the characteristic points that are easy to distinguish from nearby pixels and shifting the targeted picture in the analyzing area slightly, it is possible to detects and memorize the points where the sum of squared difference (SSD) becomes bigger than for other points. The $S S D$ is defined as shown in (1) and (2) when shifting the picture by $\Delta v$, which has sufficiently small values in window $W$, where the center is point $p$.

$$
\begin{aligned}
& S S D=S_{(p)}=\sum_{\mathrm{q} \in \mathrm{W}}^{n}(I(q)-I(q+\Delta v))^{2} \\
& S(p)=X q \in W(I(q)-I(q+\Delta v))^{2}
\end{aligned}
$$

Furthermore, to execute Taylor developing and primary approximation about the pixels of the shifted picture, $I(q+\Delta v)$ can be expressed as shown in (3) using $I(q)$ 's partial differential based on the X- and Y-axes $(I x(q)$ and $I y(q)$, respectively).

$$
I(q+\Delta v) \fallingdotseq I(q)+\left[I_{x}(q) \cdot I_{y}(q)\right] \Delta v
$$

These two equations can be combined so that $S(p)$ is expressed as shown in (4).

$$
S_{(p)}=\Delta v^{\mathrm{T}}\left[\begin{array}{cc}
\sum_{W} I_{x}^{2} & \sum_{W} I_{x} I_{y} \\
\sum_{W} I_{x} I_{y} & \sum_{W} I_{y}^{2}
\end{array}\right] \Delta v=\Delta v^{\mathrm{T}}
$$

The matrix $H(p)$ presents the features of luminance distribution around point $p$. In the theories of Harris and Shi-Tomasi, the feature amount $M$ from the characteristic value of matrix in (4) is defined as shown in (5), and is used for detecting the feature points.

$$
M=\min \left(\lambda_{1}, \lambda_{2}\right.
$$

By contrast, using the theory of Harris, we define the characteristic value of the matrix as in (6) as $\lambda_{1}$ and $\lambda_{2}$, with $\kappa$ as a parameter. When both $\lambda_{1}$ and $\lambda_{2}$ are sufficiently large, the appropriate feature points are defined as one. Then, the Harris operator can detect the specific points that make the value of $M$ in (6) sufficiently large. 


$$
M=\lambda_{1} \cdot \lambda_{2}-\kappa\left(\lambda_{1}+\lambda_{2}\right)^{2}=\operatorname{det}(H)-\kappa \cdot \operatorname{trace}^{2}(H)
$$

We used the Shi-Tomasi method in this study because the corner detection is related to its precision and burden (time and effort); whereas, with the Harris operator, the program simply judges the corners from the balance between the value of M and threshold $\kappa$. In almost all cases, the value of $\kappa$ must be obtained by performing a sufficient number of experiments. We used the Kanade method and optical flow to execute the automatic and manual searching for characteristic points in directed pictures, which are currently strong and distinguished methods in this scientific field. The outline of the procedure is as follows: (1) Determine the optical flow vectors using the least squares method, (2) identify the differences between the real values and the estimated solutions, and (3) access the estimated solutions to the real values using the hill climbing method.

The flow of the Kanade method involves creating a smoothing filter to cover (or wrap) pictures $H$ and $I$, prune the pixels, and make the new pictures $H_{1}$ and $I_{1}$, such that the resolution is $1 / 2$ (the number of pixels is $1 / 4$; the pixel size is doubled). The procedure is repeated to make $H_{2}, I_{2}, H_{3}, I_{3} \ldots H_{\mathrm{n}}, I_{\mathrm{n}}$. The "pyramids of picture" are developed based on $H$ and $I$ until the pixel size in these pictures becomes larger than the maximum amount of "travel distance" (the length of move). As was the case in prior processing, the approximate solutions of travel distance are set as $u=0$ and $v=0$, and the rank of pictures is $i=n$ according to all pixels. Third, we calculate each value based on the aforementioned methods as a loop of processions.

Next, we calculate the value of $\Delta u$ and $\Delta v$ for $H_{i}$ and $I_{i}$, the values of the travel distance $\left((u, v)=\left(u+\Delta u, v^{\prime}+\right.\right.$ $\Delta v)$ ) for each pixel, and the approximate values of travel distance $\left(u,{ }^{\prime} v\right)$ for the one-rank higher picture $\left(H_{i}-1, I_{i}-1\right)$ about the resolution using the methods below. The pixel-points $(2 u, 2 v)$ are set (the number of this picture's pixels is $(x / 2 \times y / 2))$; the $x$ and $y$ are both odd numbers, and the approximate traveling distance is $I=\left(u^{\prime}, v^{\prime}\right)$. Based on the other pixels, we define the average values of travel as $\left(u^{\prime}, v^{\prime}\right)$ for only those pixels of the four sides (upper, lower, right, and left sides), which are odd numbers. In short, it is a twin linear complement. Next, we subtract one from the current $i$ number (in the program code, $i=i-1$ ) and repeat the procedure until the value of $i$ reaches zero. We set the values of $u$ and $v$ based on $u^{\prime}=2 u$ and $v^{\prime}=2 v$, and the values of $\Delta u$ and $\Delta v$ based on $u^{\prime \prime}=2\left(u^{\prime}+2 \Delta u\right)$ and $v^{\prime \prime}=2\left(v^{\prime}+2 \Delta v\right)$, and we repeat the sequences from $i=n$ to $i=0$ in the program, while decreasing the value of $i$ by one in each iteration.

As for (4), using the theory of motion template, we successively update the motion history image and process the obtained visual data for various motion analyses and object tracking tasks using a "moving silhouette" contained in OpenCV. For clarification, we describe this feature and its peripheral functions citing the information in the OpenCV website (http://opencv.org/). First, we used a function named updateMotionHistory from the $\mathrm{C}++$ version. By estimating and indicating these arguments, these functions can be handled flexibly using the following arguments: (1) silhouette-a silhouette mask that has non-zero pixels where the motion occurs; (2) mhi-a motion history image, which is updated by the function (single-channel, 32-bit floating-point); (3) timestamp—-the current time in milliseconds or other units; and (4) duration-the maximal duration of the motion track in the same units as timestamp. The function updates the motion history image as follows:

$$
\operatorname{mhi}(x, y)= \begin{cases}\text { timestamp } & \text { if } \operatorname{silhouette}(x, y) \neq 0 \\ 0 & \text { if } \operatorname{silhouette}(x, y)=0 \text { and mhi }<\text { (timestamp - duration }) \\ \operatorname{mhi}(x, y) & \text { otherwise }\end{cases}
$$


Equation (7) shows that the mhi pixels, where the motion occurs, are set to the current timestamp, whereas the pixels where the motion last happened, a long time ago, are cleared. The function, with two important functions-calcMotionGradient and calcGlobalOrientation-implements a motion template technique. We selected and executed (1) a calculation and drawing of the main shaft of the subject, (2) manually setting and chasing the characteristic points of the subject, and (3) automatically setting and locating the characteristic point. Next, we analyzed each timeline's numerical data using significant mathematical (mainly statistical) methodologies.

Using a common, middle class spec digital camera and the latest OpenCV libraries, we calculated and drew the main shaft of the subject in each frame of obtained visual data. Second, we set the characteristic point - the right crest of the iliac bone-by manually clicking the location with a mouse, and the indicated point was located automatically using our original program (as described later). Third, using our original program, we set 24 characteristic points automatically and erased 23 of those points manually by clicking them with a mouse, leaving one point on the right crest of iliac bone.

\section{Results}

The results of this study were analyzed and discussed based on the findings of previous studies. Fig. 3-6 showed examples of the visual data obtained from the cameras, and Fig. 7-10 showed the related average data [5], [8], [10], [14], [21]-[23]. The findings indicate that the systems used are appropriate for our environment of Visual C++ Express 2010 for AVI styled video data. Fig. 3 and 4 are typical of the frames that were processed using one function to calculate the coordinates of the main shaft and the differences relating to two successive frames of visual data. Fig. 5 and 6 show the same for locating and drawing the characteristic points. Realistically, delays typically occur in the drawing speed of the shaft in the small window of Visual C++ environment; however, when computing the characteristic points, a delay was not observed.

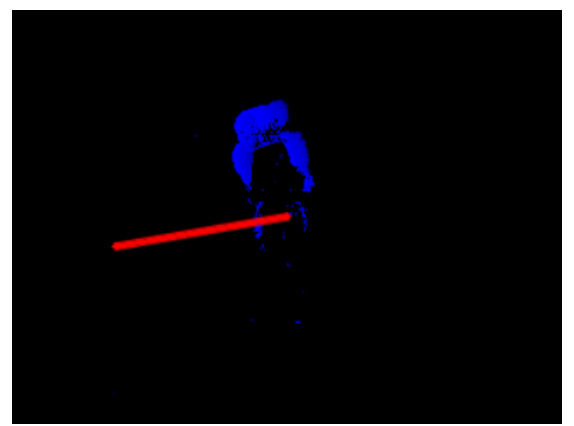

Fig. 3. Differences between pixels of two successive frames of video data obtained from the camera in front of the subject with the main shaft drawn as a red line.

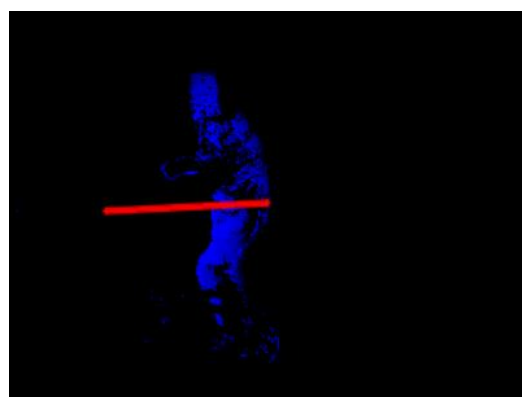

Fig. 4. Differences between pixels of two successive frames of video data obtained from the camera on the left side of the subject with the main shaft drawn as a red line. 


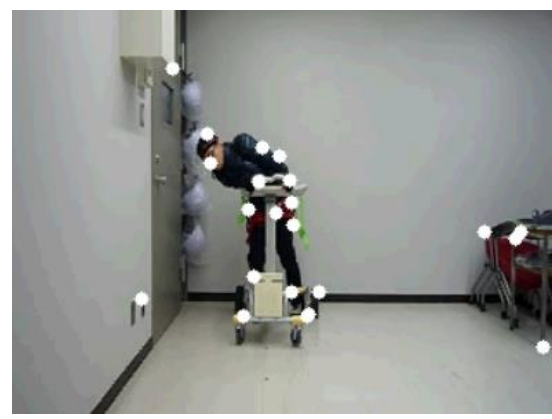

Fig. 5. Twenty-four characteristic points drawn automatically on the visual data obtained from the camera positioned in front of the subject.

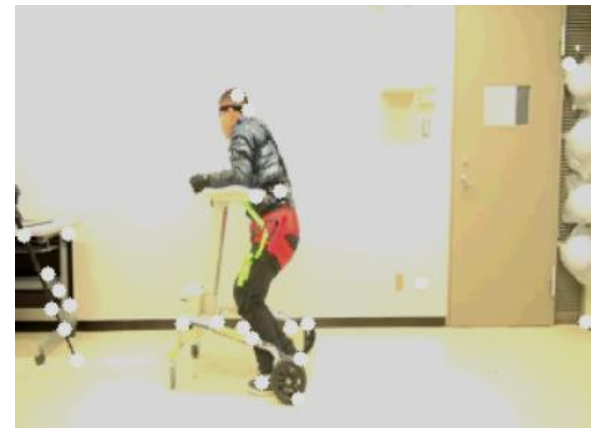

Fig. 6. Twenty-four characteristic points drawn automatically on the visual data obtained from the camera positioned on the left side of the subject.

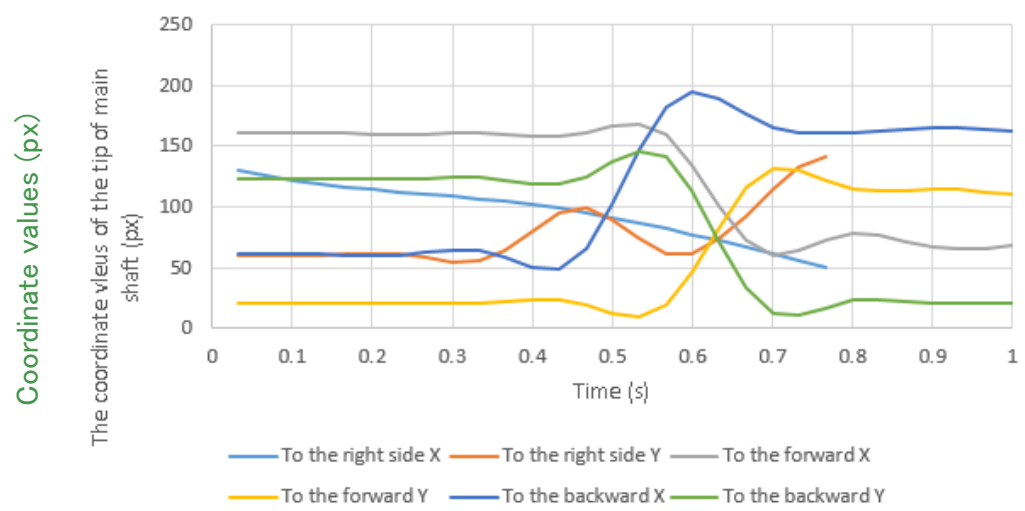

Fig. 7. Timeline chart (spline interpolation was applied) of the tip of the main shafts (the average of five trials) for the falling subject captured by the left camera.

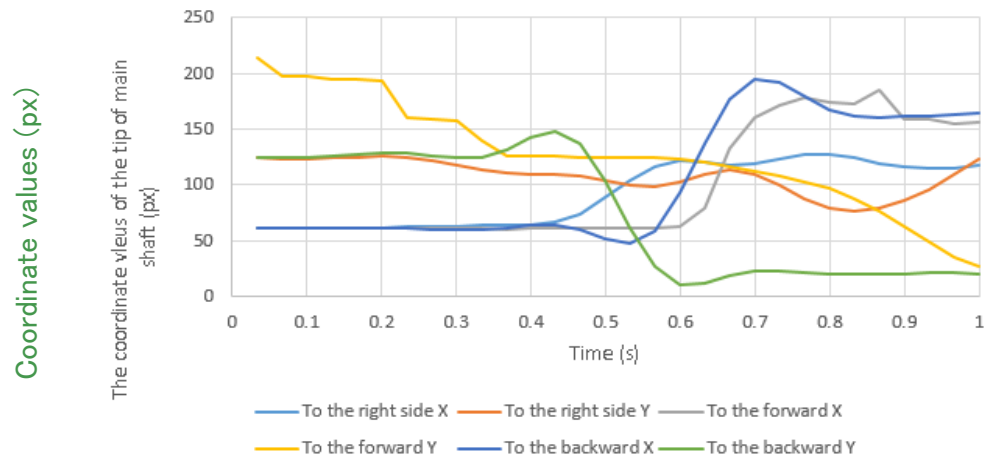

Fig. 8. Timeline chart (spline interpolation was applied) of the tip of main shafts (the average of five trials) for the falling subject captured by the front camera. 


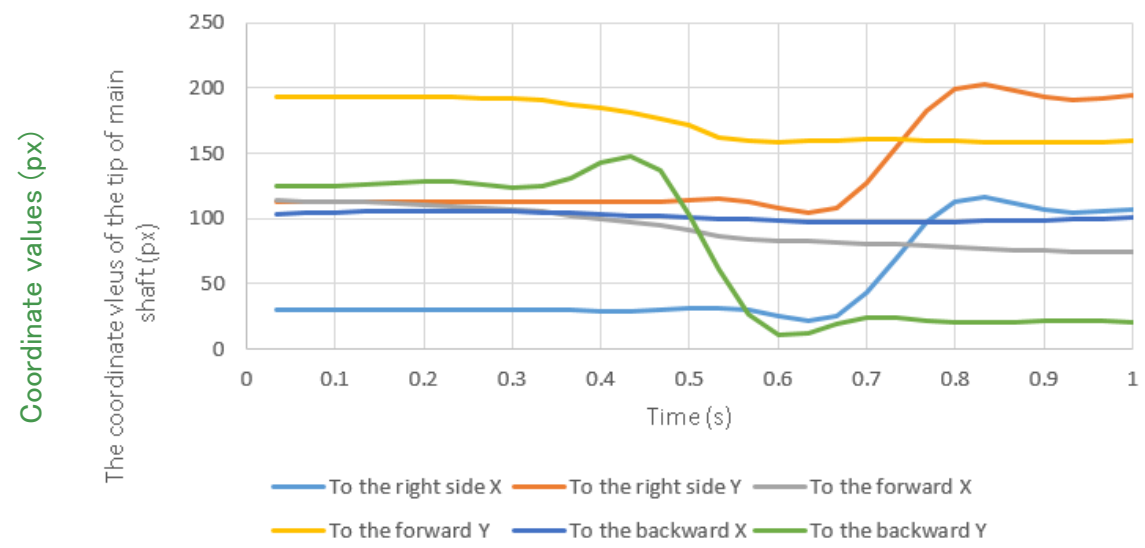

Fig. 9. Timeline chart (spline interpolation was applied) concerning coordinate values of automatically set characteristic points on top of the subject's head for the falling subject captured by the left camera.

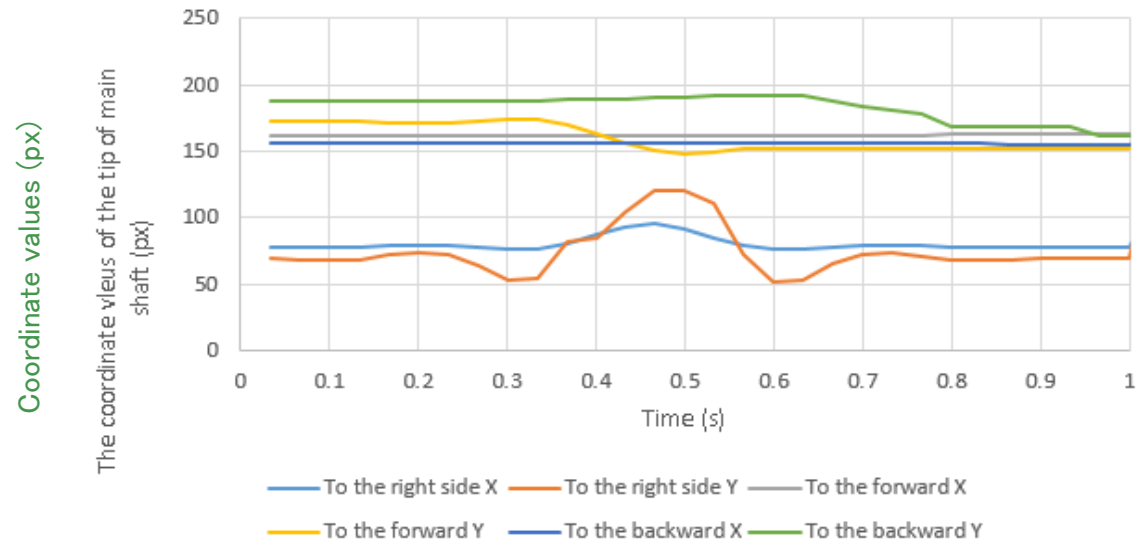

Fig. 10. Timeline chart (spline interpolation was applied) concerning coordinate values of automatically set characteristic points on top of the subject's head for the falling subject captured by the front camera.

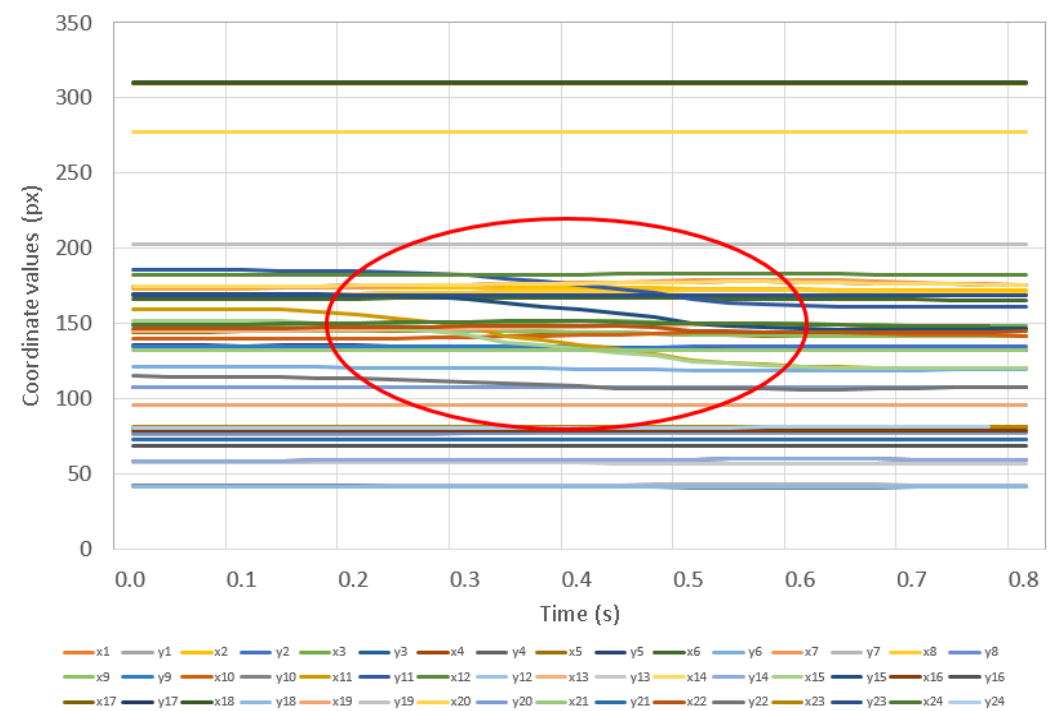

Fig. 11. The average timeline data charts showing coordinate values of automatically set 24 characteristic points on the subject falling to his right side captured by the front camera.

To assess the subject when walking, we calculated the averaged timeline data of five trials for each indicator, and to examine the subject when falling, we calculated the average data after unifying the peak points of the graphs. Then, another program was used to indicate, mark, and erase the aforementioned 
characteristic points automatically and by mouse-clicking. After marking 24 points automatically, we traced them (Fig. 5 and 6). The features and accuracy of the characteristic points' setting were inputted automatically, and we accumulated sequential timeline data for the coordinate values of the X-and Y-axes of the one point in two-dimensional geometry. Those timeline data appeared to indicate the subject's dynamism and their motion characteristics. However, other elements (e.g., biomechanical forces) could show the characteristics of the subject's movements, according to their physical attributes.

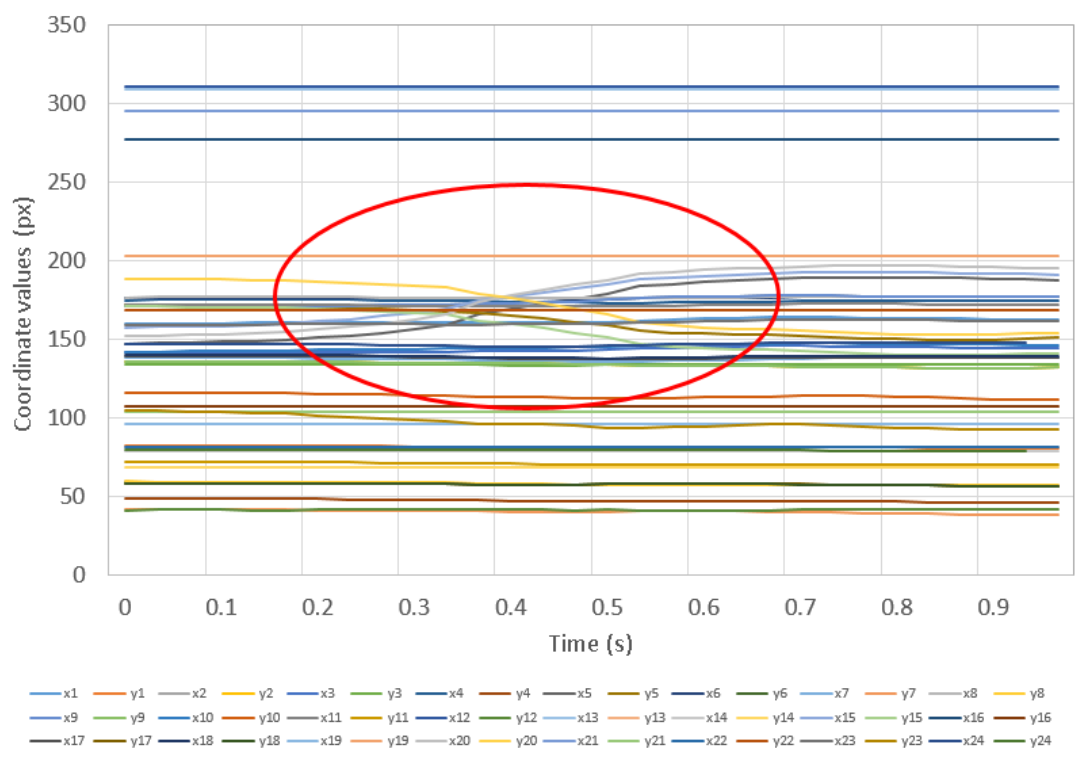

Fig. 12. The average timeline data charts showing coordinate values of 24 automatically set characteristic points on the subject falling to his left side captured by the front camera.

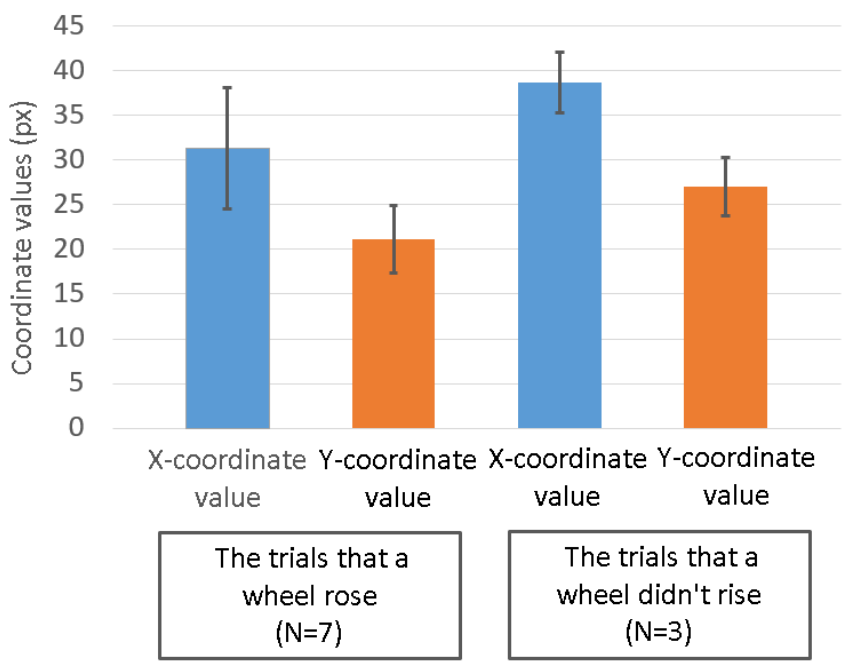

Fig. 13. Contrasting data chart showing coordinate values of automatically set characteristic points on top of the subject's head when falling to his right and left sides captured by the front camera.

\section{Discussion}

Fig. 7 and 8 show the coordinate values (according to the $\mathrm{X}$ - and $\mathrm{Y}$-axes) of the timeline chart (spline interpolation was applied) of the tip of the main shaft (average of five trials) when the subject fell, captured by the left and front cameras. Fig. 9 and 10 show the automatically set characteristic points on top of the 
subject's head when falling, captured by the left and front cameras. Fig. 11 and 12 show the average timeline data charts concerning the coordinate values of 24 automatically set characteristic points on the subject fell to his right and left sides captured by the front camera. The data chart can be compared with that of the automatically set characteristic points on top of the subject's head when falling to his right and left sides captured by the front camera to examine whether the wheels of the support system rose off of the floor or not (Fig. 13). We found clear evidence of the curves changing in relation to the previously defined main shaft and characteristic points on top of the subject's head. However, it is difficult to interpret those numerical features because of the methodological limitations and the complexities of the forms of the curves. Nevertheless, we consider that the proposed sensing methodologies, viewpoints, and concepts could be used in practical caregiving and physical training fields. The data shown in Fig. 13 can be used to check the stability of the walking support system and the suitability of the system's settings (e.g., wait-balance, size, and the users' muscles).

\section{Conclusion}

After selecting the walking system, measurement techniques and analysis methods, we measured and estimated the superficial characteristics relating to the users. Our results indicated promising physical indicators of using OpenCV-based Visual C++ programs at research institutes, academic institutions, and hospitals. These results could assist researchers, physically handicapped persons, and care workers to overcome difficulties experienced in obtaining comprehensive measurements on human bodies and systems. Additionally, we confirmed and reconsidered various measurement and performance limitations, especially regarding the distance and width concerning obtaining visual data using non-specific cameras. Overall, we obtained meaningful results and consequent benefits from this study, specifically related to users' falling. The data obtained were similar to those of past studies. Our future studies will determine the validity, durability, precision, and social impact of our vision-based methods of analysis. Subsequent studies will aim to obtain a greater variety of data from a larger number of subjects and focus on additional forces targeting other ages, races, and movements to facilitate comparisons with our data in this study. Future studies should also investigate other analysis methodologies, specific conditions of subjects, and experimental spaces. The proposed analysis systems could enhance the security and usability of the system, and contribute to the development of the next generation of machines. Future studies should also measure the system's and the users' dynamic and static properties (i.e., additional forces, those equations), as well as its measurement distant, superficial visual data measuring, and analysis. The complexity of the human body's structure has caused difficulties when taking measurements and understanding the different responses between individuals, thus impeding our understanding of various physical data. Our data therefore indicate a promising outlook for developing practical support for physically challenged and elderly people.

\section{Acknowledgment}

Our heartfelt appreciation goes to colleagues at the National Institute of Advanced Industrial Science and Technology (AIST), members of Impulsing Paradigm Change through Disruptive Technologies (ImPACT) Program/Cabinet Office, Government of Japan, for their considerable support, feedback, and valuable comments.s

\section{References}

[1] Wei, Y., Xiangxin, K., Lee, \& M. C. (2014). A new vision and navigation research for a guide-dog robot system in urban system. Proceeding of IEEE/ASME International Conference on Advanced Intelligent 
Mechatronics (AIM) 2014.

[2] Payam, N., et al. (2015). Indoor positioning of a robotic walking assistant for large public environments. IEEE Transactions on Instrumentation and Measurement, 64(11), 2965-2976.

[3] Satoshi, M., \& Kaori, F. (2011). Stabilization of projected image for wearable walking support system using pico-projector. Proceedings of IEEE 17th International Conference on Embedded and Real-Time Computing Systems and Applications (RTCSA) 2011, 113-116.

[4] Kabalan, C., \& Badr, G. (2016). Computer vision guidance system for indoor navigation of visually impaired people. The proceeding of 2016 IEEE 8th International Conference on Intelligent Systems (IS), 449-454.

[5] Augustyniak, P., Smoleń, M., Mikrut, Z., \& Kańtoch, E. (2014). Seamless tracing of human behavior using complementary. Sensors, 14(5), 7831-7856.

[6] Valls, M. J., Poon, J., \& Huang, S. (2012). Low-cost visual tracking with an intelligent wheelchair for innovative assistive care. Control Automation Robotics \& Vision (ICARCV), 1540-1545.

[7] Dulari, S. (2016). Automatic camera based eye controlled wheelchair system using raspberry Pi. International Journal of Science, Engineering and Technology Research (IJSETR), 5(1).

[8] Machida, E., et al. (2012). Human motion tracking of mobile robot with Kinect 3D sensor. Proceedings of IEEE SICE Annual Conference 2012, 2207-2211.

[9] Said, A. M., et al. (2013). Real-time vision based dynamic sinkage detection for exploration rovers. Proceedings of 2013 IEEE/RSJ International Conference on Intelligent Robots and Systems (IROS).

[10] Kuo-Lan, S., Li, B. Y., \& Kuo-Hsien, H. (2015). Development of tracking system for mobile robot using image recognition algorithms. Transactions of the Canadian Society for Mechanical Engineering, 39(3), 501-513.

[11] Han, S., Zhang, Q., Nic, B., \& Reid, J. F. (2004). A guidance directrix approach to vision-based vehicle guidance systems. Computers and Electronics in Agriculture, 43, 179-195.

[12] Mehta, S. S., \& Burks, T. F. (2014). Vision-based control of robotic manipulator for citrus harvesting. Computers and Electronics in Agriculture, 102, 146-158.

[13] Zhou, F., De la Torre, F., \& Hodgins, J. K. (2013). Hierarchical aligned cluster analysis for temporal clustering of human motion. IEEE Trans. on Pattern Analysis and Machine Intelligence, 35(3), 582-596.

[14] Mukhopadhyay, S. C. (2015). Wearable sensors for human activity monitoring. A Review: IEEE Sensors Journal, 15(3), 1321-1330.

[15] Chen, Z. (2009). Efficient block matching algorithm for motion estimation. International Journal of Signal Processing, 5(2), 133-137.

[16] Kuwai, H., Nagata, N., \& Toyosawa, S. (2014). Jissen OpenCV 2.4 for Python. Tokyo: CUTT SYSTEM.

[17] Nagata, N., \& Toyosawa, S. (2009). Jissen OpenCV 2.4. Tokyo: CUTT SYSTEM.

[18] Nagata, N., \& Toyosawa, S. (2012). Digital Eizou Nyumon. Tokyo: CUTT SYSTEM.

[19] Kawakura, S., \& Shibasaki, R. (2013). Supporting systems for agricultural worker's skill and security. Proceedings of Asian Conference Remote Sensing (ACRS), 71-77.

[20] Kawakura, S., \& Shibasaki, R. (2014). Timeline effects of vocal instructions from computer programs on agricultural technical teaching. Journal of Advanced Agricultural Technologies, 1(2), 104-112.

[21] Murao, T., Hirao, Y., \& Hashimoto, H. (2011). Skill level evaluation for Taijiquan based on curve fitting and logarithmic distribution diagram of curvature. SICE Journal of Control, Measurement, and System Integration, 4(1), 1-5.

[22] Kan, T. (2013). Excel de Manabu Toukei kaiseki Nyuumon. Tokyo: Ohmsha.

[23] Kan, T. (2014). Excel de Manabu Toukeiteki Yosoku. Tokyo: Ohmsha. 


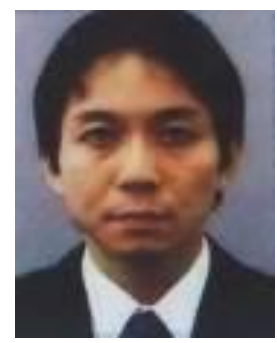

Shinji Kawakura works in research associate at National Institute of Advanced Industrial Science and Technology (AIST)/Department of Information Technology and Human Factors, received the Ph.D. in Environmentology, the University of Tokyo, 2015.

He was born on July 14, 1978. He get the B.A in control system engineering, Tokyo Institute of Technology, 2003; the M.A. in human-factors engineering, Tokyo Institute of Technology, 2005. 